International Journal of Pure and Applied Mathematics

Volume 92 No. 4 2014, 609-618

ISSN: 1311-8080 (printed version); ISSN: 1314-3395 (on-line version)

url: http://www.ijpam.eu

doi: http://dx.doi.org/10.12732/ijpam.v92i4.16

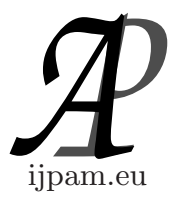

\title{
PSI-ORDINARY DICHOTOMY OF THE SOLUTIONS OF IMPULSIVE DIFFERENTIAL EQUATIONS \\ IN A BANACH SPACE
}

\author{
Stoyan Zlatev \\ Faculty of Mathematics and Informatics \\ University of Plovdiv \\ 236, Bulgaria Blvd., 4003 Plovdiv, BULGARIA
}

\begin{abstract}
In the paper a dependence is established between the $\psi$-ordinary dichotomy of a homogeneous impulsive differential equation in a Banach space and the existence of $\psi$-bounded solution of the appropriate nonhomogeneous equation.
\end{abstract}

AMS Subject Classification: 34A37, 34G10, 34D09

Key Words: ordinary dichotomy for impulsive differential equations, $\psi$ dichotomy, $\psi$-boundedness

\section{Introduction}

The mathematical theory of impulsive differential equations is much richer in problems in comparison with the corresponding theory of ordinary differential equations. That is why the impulsive differential equations are adequate apparatus for mathematical simulation of numeruous processes and phenomena which are studied in biology, phisics and technology.

The problem of $\psi$-boundedness and $\psi$-stability of the solutions of differential equations in finite dimensional Euclidean spaces has been studied by many authors, as e.q. Akinyele [1], Constantin [7]. In these papers, the function

Received: February 3, 2014

(c) 2014 Academic Publications, Ltd. url: www.acadpubl.eu 
$\psi$ is a scalar continuous function (and increasing, differentiable and bounded in [1], nondecreasing and such that $\psi(t) \geq 1$ on $R_{+}$in [7]). In the papers of Diamandescu [9],[10],[11] and Boi [3],[4],[5] $\psi$ is a nonnegative continuous diagonal matrix function.

Inspired by the famous monographs of Coppel [6], Daleckii and Krein [8] and Massera and Schaeffer [15], where the important notion of exponential and ordinary dichotomy is considered in details, Diamandescu [9]-[11] and Boi [3][5] introduced and studied the $\psi$-dichotomy for linear differential equations in finite dimensional Euclidean space. The concept of $\psi$-dichotomy for arbitrary Banach spaces is studied in [13] and [14]. In this case $\psi(t)$ is an arbitrary bounded invertible linear operator, instead of the restriction to be a nonnegative diagonal matrix.

The goal of the present paper is to etablish a dependence between the $\psi$-ordinary dichotomy of a homogeneous impulsive equation in a Banach space and the existence of a $\psi$-bounded on the semi-axis solution of the corresponding nonhomogeneous impulsive equation.

\section{Preliminaries}

Let $X$ be an arbitrary Banach space with norm |.| and let $L B(X)$ be the space of all linear bounded operators acting in $X$ with the norm $\|$.$\| and identity I$. Denote $R_{+}=[0, \infty)$.

We consider the nonhomogenous impulsive equation

$$
\begin{gathered}
\frac{\mathrm{d} x}{\mathrm{~d} t}=A(t) x+f(t) \quad\left(t \neq t_{n}\right) \\
x\left(t_{n}+0\right)=Q_{n} x\left(t_{n}\right)+h_{n} \quad(n=1,2,3, \ldots)
\end{gathered}
$$

where the operator valued function $A():. R_{+} \rightarrow L B(X)$ and the function $f():. R_{+} \rightarrow X$ are strong measurable and Bochner integrable on the finite subintervals of $R_{+}, Q_{n} \in L B(X)(n=1,2,3, \ldots), T=\left\{t_{n}\right\}_{n=1}^{\infty}$ is a sequence of points on the semi-axis $R_{+}$satisfying the condition

$$
0<t_{1}<t_{2}<\ldots, \lim _{n \rightarrow \infty} t_{n}=\infty .
$$

The corresponding homogenous impulsive equation is

$$
\begin{gathered}
\frac{\mathrm{d} x}{\mathrm{~d} t}=A(t) x \quad\left(t \neq t_{n}\right) \\
x\left(t_{n}+0\right)=Q_{n} x\left(t_{n}\right) \quad(t=1,2,3, \ldots) .
\end{gathered}
$$


By a solution of the impulsive equation (1), (2) (or (3), (4)) we will understand a piecewise continuous function $x(t)$ with points of discontinuity of first kind $t_{1}, t_{2}, \ldots$ which satisfies (1) (or (3)) for $t \neq t_{n}$ and (2) (or (4)) otherwise.

Let $R L(X)$ be the subspace of all invertible operators in $L B(X)$ and let $\psi():. R_{+} \rightarrow R L(X)$ be a continuous for any $t \in R_{+}$operator-function.

Definition 1. A function $u():. R_{+} \rightarrow X$ is said to be $\psi$-bounded on $R_{+}$ if $\psi(t) u(t)$ is bounded on $R_{+}$.

A function $f():. R_{+} \rightarrow X$ is said to be $\psi$-Bochner integrable on $R_{+}$if it is measurable and $\int_{0}^{\infty}|\psi(\tau) f(\tau)| d \tau<\infty$.

Let $C_{\psi}(X, T)$ denote the space of all $\psi$-bounded on $R_{+}$functions with values in $X$ which are continuous for $t \neq t_{n}$, have discontinuities of the first kind for $t=t_{n}$ and are continuous from the left for $t=t_{n}$, which is a Banach space with the norm

$$
\||| f||_{C_{\psi}}=\sup _{t \in R_{+}}|\psi(t) f(t)| .
$$

Let $L_{\psi}(X)$ denote the Banach space of all $\psi$-Bochner integrable on $R_{+}$ functions with values in $X$ with the norm

$$
\||| f||_{L_{\psi}}=\int_{0}^{\infty}|\psi(s) f(s)| \mathrm{d} s .
$$

Let $H_{\psi}$ denote the set

$$
H_{\psi}(X)=\left\{h=\left\{h_{n}\right\}_{n=1}^{\infty}: \sum_{j=1}^{\infty}\left|\psi\left(t_{j}+0\right) h_{j}\right|<\infty\right\}
$$

which is also a Banach space with respect to the norm

$$
\left.|||h|\right|_{H_{\psi}}=\sum_{j=1}^{\infty}\left|\psi\left(t_{j}+0\right) h_{j}\right| .
$$

Definition 2. The homogenous impulsive equation (3), (4) is said to be $\psi$-ordinary dichotomous on $R_{+}$if there exist a pair $P_{1}$ and $P_{2}=I-P_{1}$ mutually complementary projections in $X$ and a number $M>0$ for which the inequalities

$$
\begin{array}{cc}
\left\|\psi(t) V(t) P_{1} V^{-1}(s) \psi^{-1}(s)\right\| \leq M & (0 \leq s \leq t<\infty), \\
\left\|\psi(t) V(t) P_{2} V^{-1}(s) \psi^{-1}(s)\right\| \leq M & (0 \leq t \leq s<\infty)
\end{array}
$$

hold, where $V(t)=V(t, 0)$ and $V(t, s)(0 \leq s, t<\infty)$ is the Cauchy evolutionary operator $[16,12]$ of the impulsive equation (3), (4). 
We shall say that condition $(\mathrm{H})$ is satisfied if the following conditions hold:

H1) The operator-valued function $A():. R_{+} \rightarrow L B(X)$ is continuous.

H2) $Q_{n} \in R L(X) \quad(n=1,2, \ldots)$.

\section{Main Results}

Theorem 1. Let the following conditions hold:

1. Condition $(H)$ is satisfied.

2. Equation (3), (4) is $\psi$-ordinary dichotomous.

Then for any function $f \in L_{\psi}(X)$ and any sequence $h \in H_{\psi}(X)$ there exists a solution of the nonhomogeneous equation (1), (2) which is $\psi$-bounded on $R_{+}$.

Proof. Consider the function

$$
\begin{aligned}
\tilde{x}(t) & =\int_{0}^{t} \psi(t) V(t) P_{1} V^{-1}(s) f(s) \mathrm{d} s-\int_{t}^{\infty} \psi(t) V(t) P_{2} V^{-1}(s) f(s) \mathrm{d} s+ \\
& +\sum_{t_{j}<t} \psi(t) V(t) P_{1} V^{-1}\left(t_{j}+0\right) h_{j}-\sum_{t_{j} \geq t} \psi(t) V(t) P_{2} V^{-1}\left(t_{j}+0\right) h_{j}= \\
& =\int_{0}^{t} \psi(t) V(t) P_{1} V^{-1}(s) \psi^{-1}(s) \psi(s) f(s) \mathrm{d} s- \\
& -\int_{t}^{\infty} \psi(t) V(t) P_{2} V^{-1}(s) \psi^{-1}(s) \psi(s) f(s) \mathrm{d} s+ \\
& +\sum_{t_{j}<t} \psi(t) V(t) P_{1} V^{-1}\left(t_{j}+0\right) \psi^{-1}\left(t_{j}+0\right) \psi\left(t_{j}+0\right) h_{j}- \\
& -\sum_{t_{j} \geq t} \psi(t) V(t) P_{2} V^{-1}\left(t_{j}+0\right) \psi^{-1}\left(t_{j}+0\right) \psi\left(t_{j}+0\right) h_{j}
\end{aligned}
$$


We shall estimate the norm of $\tilde{x}(t)$ :

$$
\begin{aligned}
|\tilde{x}(t)| \leq & \int_{0}^{t}\left\|\psi(t) V(t) P_{1} V^{-1}(s) \psi^{-1}(s)\right\||\psi(s) f(s)| \mathrm{d} s+ \\
& +\int_{t}^{\infty}\left\|\psi(t) V(t) P_{2} V^{-1}(s) \psi^{-1}(s)\right\||\psi(s) f(s)| \mathrm{d} s+ \\
& +\sum_{t_{j}<t}\left\|\psi(t) V(t) P_{1} V^{-1}\left(t_{j}+0\right) \psi^{-1}\left(t_{j}+0\right)\right\|\left|\psi\left(t_{j}+0\right) h_{j}\right|+ \\
+ & \sum_{t_{j} \geq t}\left\|\psi(t) V(t) P_{2} V^{-1}\left(t_{j}+0\right) \psi^{-1}\left(t_{j}+0\right)\right\|\left|\psi\left(t_{j}+0\right) h_{j}\right| \leq \\
& \leq M \int_{0}^{t}|\psi(s) f(s)| \mathrm{d} s+M \int_{t}^{\infty}|\psi(s) f(s)| \mathrm{d} s+ \\
& +M \sum_{t_{j}<t}\left|\psi\left(t_{j}+0\right) h_{j}\right|+M \sum_{t_{j} \geq t}^{\infty}\left|\psi\left(t_{j}+0\right) h_{j}\right|= \\
& =M \int_{0}^{\infty}|\psi(s) f(s)| \mathrm{d} s+M \sum_{j=1}^{\infty}\left|\psi\left(t_{j}+0\right) h_{j}\right|<\infty
\end{aligned}
$$

Hence $\tilde{x}(t)$ is bounded on $R_{+}$.

Let $x(t)=\psi^{-1}(t) \tilde{x}(t)$. Obviously $x(t)$ is $\psi$-bounded on $R_{+}$. It is immediately verified that the function $x(t)$ is continuous for $t \neq t_{n}$ and that the limit values $x\left(t_{n}+0\right)(n=1,2, \ldots)$ exist. We shall show that the function $x(t)$ satisfies the impulsive equation (1), (2) using the well known equalities

$$
\frac{\mathrm{d} V(t, s)}{\mathrm{d} t}=A(t) V(t, s), \quad \frac{\mathrm{d} V(t, s)}{\mathrm{d} s}=V(t, s) A(s)
$$

and

$$
V\left(t_{n}+0, s\right)=Q_{n} V\left(t_{n}, s\right) \quad\left(0 \leq s \leq t_{n}<\infty ; n=1,2,3, \ldots\right) .
$$

We differentiate $x(t)$ for $t \neq t_{n}$ and obtain

$$
\begin{aligned}
\frac{\mathrm{d} x}{\mathrm{~d} t} & =A(t) \int_{0}^{t} V(t) P_{1} V^{-1}(s) f(s) \mathrm{d} s+V(t) P_{1} V^{-1}(t) f(t)+ \\
& +V(t) P_{2} V^{-1}(t) f(t)-A(t) \int_{t}^{\infty} V(t) P_{2} V^{-1}(s) f(s) \mathrm{d} s+ \\
& +\sum_{t_{j}<t} A(t) V(t) P_{1} V^{-1}\left(t_{j}+0\right) h_{j}-\sum_{t_{j} \geq t} A(t) V(t) P_{2} V^{-1}\left(t_{j}+0\right) h_{j}= \\
& =A(t) x(t)+V(t) P_{1} V^{-1}(t) f(t)+V(t) P_{2} V^{-1}(t) f(t)= \\
& =A(t) x(t)+f(t)
\end{aligned}
$$


Analogously for $t=t_{n} \quad(n=1,2, \ldots)$ we obtain

$$
\begin{aligned}
x\left(t_{n}+0\right) & =\int_{0}^{t_{n}} V\left(t_{n}+0\right) P_{1} V^{-1}(s) f(s) \mathrm{d} s- \\
& -\int_{t_{n}}^{\infty} V\left(t_{n}+0\right) P_{2} V^{-1}(s) f(s) \mathrm{d} s+ \\
& +\sum_{t_{j}<t_{n}} V\left(t_{n}+0\right) P_{1} V^{-1}\left(t_{j}+0\right) h_{j}- \\
& -\sum_{t_{j} \geq t_{n}} V\left(t_{n}+0\right) P_{2} V^{-1}\left(t_{j}+0\right) h_{j}= \\
= & Q_{n} \int_{0}^{t_{n}} V\left(t_{n}\right) P_{1} V^{-1}(s) f(s) \mathrm{d} s- \\
+ & Q_{n} \int_{t_{n}}^{\infty} V\left(t_{n}\right) P_{2} V^{-1}(s) f(s) \mathrm{d} s+ \\
+ & Q_{n} \sum_{t_{j}<t_{n}} V\left(t_{n}\right) P_{1} V^{-1}\left(t_{j}+0\right) h_{j}- \\
& -Q_{n} \sum_{t_{j} \geq t_{n}} V\left(t_{n}\right) P_{1} V^{-1}\left(t_{j}+0\right) h_{j}+ \\
+ & V\left(t_{n}\right) P_{1} V^{-1}\left(t_{j}+0\right) h_{j}+ \\
+ & V\left(t_{n}\right) P_{2} V^{-1}\left(t_{j}+0\right) h_{j}= \\
= & Q_{n} x\left(t_{n}\right)+h_{n}
\end{aligned}
$$

Hence the function $x(t)$ is a $\psi$-bounded solution of the nonhomogeneous equation (1), (2) on $R_{+}$.

Remark 1. For $\psi(t)=I$, i.e. for impulse equation with ordinary dichotomy, Theorem 1 is proved under weaker conditions comparing with the result obtained in [2].

Let $X_{1}$ be the linear manifold of all $\xi \in X$ for which the function $V(t) \xi(t \in$ $\left.R_{+}\right)$is $\psi$-bounded.

Lemma 1. Let the following conditions hold:

1. Condition $(H)$ is satisfied.

2. $B_{\psi}(X)$ is an arbitrary Banach space of functions $f():. R_{+} \rightarrow X$ and for any function $f \in B_{\psi}(X)$ the nonhomogeneous equation (1), (2) has at least one 
$\psi$-bounded on $R_{+}$solution $x \in C_{\psi}(X, T)$

$$
\left(|||x| \|_{C_{\psi}}=\sup _{t \in R_{+}}|\psi(t) x(t)|<\infty\right) .
$$

3. The set $X_{1}$ is a complementary subspace of $X$ and let $X_{2}$ is a complement of it $\left(X_{1}+X_{2}=X\right)$.

Then to each function $f(t) \in B_{\psi}(X)$ there corresponds a unique $\psi$-bounded on $R_{+}$solution $x(t)$ starting from $X_{2}$, i.e. $x(0) \in X_{2}$.

This solution satisfies the estimate

$$
\left\||| x||_{C_{\psi}} \leq\left. k|\| f|\right|_{B_{\psi}}\right.
$$

where $k>0$ is a constant not depending on $f$.

Proof. Let $P_{1}$ and $P_{2}$ be the mutually complementary projections on the subspaces $X_{1}$ and $X_{2}$.

Denote by $C_{\psi}^{0}(X, T)$ the subspace of $C_{\psi}(X, T)$ consisting of the functions which satisfy the condition

$$
x\left(t_{n}+0\right)-Q_{n} x\left(t_{n}\right)=0 \quad(n=1,2,3, \ldots) .
$$

It is not hard to check that if $x_{1}(t)$ and $x_{2}(t)$ are two solutions of the nonhomogeneous impulsive equation (1), (2), then their difference $z(t)=x_{1}(t)-x_{2}(t)$ is a solution of the homogeneous impulsive equation (3), (4). If the solutions $x_{1}(t)$ and $x_{2}(t)$ are $\psi$-bounded, then $z(t)$ is $\psi$-bounded too, hence $z(0) \in X_{1}$.

If $x(t)$ is a solution of (1), (2) lying in $C_{\psi}^{0}(X, T)$, then $\tilde{x}(t)=x(t)-$ $V(t) P_{1} x(0)$ is also a solution of $(1),(2)$ lying in $C_{\psi}^{0}(X, T)$ with initial value $\tilde{x}(0)=P_{2} x(0) \in X_{2}$. From the conditions of the lemma it follows that for $f(t) \in B_{\psi}(X)$ the equation (1), (2) has a solution $\tilde{x}(t) \in C_{\psi}^{0}(X, T)$ satisfying the equality $P_{1} \tilde{x}(0)=0$. But this solution is unique, since the difference of two such solutions would be a $\psi$-bounded solution of the homogeneous equation which is initially in $X_{2}$, which is possible only for the zero solution. Thus an operator $\tilde{K}: B_{\psi}(X) \rightarrow C_{\psi}^{0}(X, T)$ is defined which associates with each element $f \in B_{\psi}(X)$ a solution of equation (1), (2). From the Banach's closed graph theorem it follows that this operator is continuous, i.e. there exists a number $k$ for which

$$
|| \tilde{K} f||_{C_{\psi}^{0}} \leq k|||f| \|_{B_{\psi}} .
$$

Lemma 1 is proved. 
Theorem 2. Let the following conditions hold:

1. Condition $(H)$ is satisfied.

2. The set $X_{1}$ is a complementary subspace of $X$

3. The nonhomogeneous equation (1), (2) has a $\psi$-bounded on $R_{+}$solution for any function $f \in L_{\psi}(X)$ and $h=\left\{h_{n}\right\}_{n=1}^{\infty}=0$.

Then the homogeneous equation (3), (4) is $\psi$-ordinary dichotomous.

Proof. We shall show that the estimate $\left\|\psi(t) G(t, s) \psi^{-1}(s)\right\| \leq K$ is valid for $0 \leq s, t<\infty$, where $K$ is a constant and

$$
G(t, s)=\left\{\begin{array}{lc}
V(t) P_{1} V^{-1}(s) & (0 \leq s \leq t<\infty) \\
-V(t) P_{2} V^{-1}(s) & (0 \leq t \leq s<\infty)
\end{array}\right.
$$

For this purpose let us consider the function $f$ defined as follows

$$
f(t)= \begin{cases}\psi^{-1}(t) z & s-a \leq t \leq s \\ 0 & \text { otherwise }\end{cases}
$$

where $s \geq a>0$ are fixed points and $z \in X$ and $|z|=1$. Obviously $f \in L_{\psi}(X)$ and $\||f|\|_{L_{\psi}}=a$.

Introduce the function $x(t)$ by the formula

$$
x(t)=\int_{s-a}^{s} G(t, \tau) \psi^{-1}(\tau) z \mathrm{~d} \tau .
$$

For $t \geq a$ the equality $x(t)=V(t) \xi$ holds, where

$$
\xi=\int_{s-a}^{s} P_{1} V^{-1}(\tau) \psi^{-1}(\tau) z \mathrm{~d} \tau \in X_{1}
$$

i.e. the function $x(t)$ is $\psi$-bounded. For $0 \leq t \leq a$ the function $x(t)$ is also $\psi$-bounded, hence it is $\psi$-bounded on the whole semi-axis $R_{+}$. Moreover

$$
x(0)=\int_{s-a}^{s} G(0, \tau) \psi^{-1}(\tau) z \mathrm{~d} \tau=-P_{2} \int_{s-a}^{s} V^{-1}(\text { tau }) \psi^{-1}(\tau) z \mathrm{~d} \tau \in X_{2} .
$$

Since $x(t)$ is obviously a $\psi$-bounded solution, from Lemma 1 it follows that $x(t)$ is the unique $\psi$-bounded on $R_{+}$solution of (1), (2):

$$
x(t)=\int_{0}^{\infty} G(t, \tau) f(\tau) \mathrm{d} \tau=\int_{s-a}^{s} G(t, \tau) \psi^{-1}(\tau) z \mathrm{~d} \tau
$$


and there exists a constant $k>0$ for which the followings estimate holds

$$
\left.|||x|\right|_{C_{\psi}} \leq\left. k|\| f|\right|_{B_{\psi}},
$$

i.e.

$$
|\psi(t) x(t)|=\left|\int_{s-a}^{s} \psi(t) G(t, \tau) \psi^{-1}(\tau) z \mathrm{~d} \tau\right| \leq k a .
$$

It follows that

$$
\left|\frac{1}{a} \int_{s-a}^{s} \psi(t) G(t, \tau) \psi^{-1}(\tau) z \mathrm{~d} \tau\right| \leq k .
$$

Let $a \rightarrow 0$. Then the following inequality holds

$$
\left|\psi(t) G(t, \tau) \psi^{-1}(\tau) z\right| \leq k \quad(|z|=1),
$$

hence

$$
\left\|\psi(t) G(t, \tau) \psi^{-1}(t)\right\| \leq k \quad(0 \leq s, t<\infty) .
$$

Theorem 2 is proved.

\section{References}

[1] O. Akinyele, On partial stability and boundedness of degree k, Atti. Accad. Naz. Lincei Rend. Cl. Sci. Fis. Mat. Natur. 8, 65 (1978), 259-264.

[2] D. Bajnov, S. Kostadinov, P.Zabrejko, Dichotomy of the solutions of impulse differential equations in a Banach space, Math. Rep. Toyama Univ., 12 (1989), 159-166.

[3] P. Boi, On the $\psi$-dichotomy for homogeneous linear differential equations, Electron. J. Differ. Equ., 2006, No. 40, 1-12.

[4] P. Boi, Existence of $\psi$-bounded solutions for nonhomogeneous linear differential equations, Electron. J. Differ. Equ., 2007, No. 52, 1-10.

[5] P. Boi, Behavior at infinity of psi-evanescent solutions to linear differential equations, Electron. J. Differ. Equ., 2010, No. 102, 1-9.

[6] W. Coppel, Dichotomies in stability theory, Lectures Notes in Mathematics, Vol. 629, Springer Verlag, Berlin (1978).

[7] A. Constantin, Asymptotic properties of solutions of differential equations, Analele Universitatii of Timisoara, Seria Stiinte Matematice bf 30 (1992), 183-225. 
[8] J. Daleckii, M. Krein, Stability of Solutions of Differential Equations in Banach space, American Mathematical Society, Providence, Rhode Island (1974).

[9] A. Diamandescu, Note on the $\Psi$-boundedness of the solutions of a system of differential equations, Acta Math. Univ. Comen., New Ser. 73 (2004), No. 2, 223-233.

[10] A. Diamandescu, Existence of $\psi$-bounded solutions for a system of differential equations, Electron. J. Differ. Equ., 2004, No. 63, 1-6.

[11] A. Diamandescu, Existence of $\Psi$-bounded solutions for nonhomogeneous linear difference equations, Appl. Math. E-Notes, 10 (2010), 94-102.

[12] A. Georgieva, H. Kiskinov, Some properties of the characteristic exponents of impulse differential equations in a Banach space, Scientific Works, Plovdiv University, 37 (2010), book 3, Mathematics, 35-44.

[13] A. Georgieva, H. Kiskinov, S. Kostadinov, A. Zahariev, Psi-exponential dichotomy for linear differential equations in a Banach space, Electron. J. Differ. Equ., 2013, No. 153, 1-13.

[14] A. Georgieva, H. Kiskinov, S. Kostadinov, A. Zahariev, Existence of solutions of nonlinear differential equations with Psi-exponential or Psiordinary dichotomous linear part in a Banach space, Electron. J. Qual. Theory Differ. Equ., 2014, No. 2, 1-10.

[15] J. Massera, J. Schaeffer, Linear Differential Equations and Function Spaces, Academic Press (1966)

[16] P. Zabrejko, D. Bajnov, S. Kostadinov, Characteristic exponents of impulsive differential equations in a Banach space, Int. J. Theor. Phys., 27 (1988), No. 6, 731-743. 